\title{
The Design and Research of the construction of Fujian Meteorological Comprehensive Management Platform
}

\author{
Zheng Wei ${ }^{1, a}$, Wu Yinan ${ }^{1, a}$ and Yu Juan ${ }^{1, a}$ \\ ${ }^{1}$ Zherong County Meteorological Bureau, China \\ azw1023@126.com
}

Keywords: E-government, Comprehensive management, Information sharing, Application support, J2EE framework.

\begin{abstract}
In order to improve the level of the meteorological administration in the province, the electronic government affairs office and the government management in the network information system are standardized. The platform integrates the multi-tier architecture, supporting WebService, SOAP, XML and other standards to carry out rapid development, especially the visualization process configuration. At the same time, the government standard working process is introduced in accordance with the situation in the province to carry out re allocation and adjustment, and it makes the flexible and efficiency in the government work standard to ensure the province's government work standardization. As a standard for information exchange, XML is the realization of the data exchange of platform and the distribution system, and then the XML data carries out encryption and decryption, to ensure the security of data transmission, the data does not occupy too much bandwidth to ensure the bandwidth efficiency for the XML data compression. It is proved by practice that the configuration of the government affair work flow solve the previous government link transfer of disorder and inefficiency, and the different work is incorporated into the assessment of management by objectives, each job is calculated to quantify by score and heterogeneous data on the platform are fully interactive, to ensure the real-time updating and advancing of e-government business.
\end{abstract}

\section{Introduction}

With the rapid development of network technology, database technology and development technology, the electronic government affairs system has realized the operation in most countries [1]. Although the Bureau started late in the development of e-government system, communication network has covered the levels of mature size from the province to county, which provides support base to achieve the province's e-government network office. On the premise of the information technology, J2EE technology and information system construction, the comprehensive management information system of Fujian meteorological bureau is very mature, and China meteorological bureau uses the E-government theory to carry out guidance for national meteorological department e-government work, and then the use of government e-government top-level design idea analyzes characteristics of the province's meteorological e-government business, this system is developed based on the research and application of role access control, combined with a large number of previous work under the J2EE [2-3]. The development of the Fujian integrated management information system, its core business platform is efficiency in order to better solve the province's meteorological department e-government management and processing capabilities, improving the traditional government processing, ensuring the safety of the government process and e-government information sharing, to better promote the provincial government management means change. And its practical significance makes the province's meteorological department to get relief from the original disordered government affairs, system functions well meet the province's meteorological department government affairs work need, heterogeneous data can also achieve transfer, the original cannot be quantified work that can be thoroughly quantized process [4]. These are based on the system of the core application supporting platform to expand, this paper mainly discusses a core application supporting platform based on J2EE architecture, and the key points are discussed in detail to elucidate the structure of the system design and the core technology application. 


\section{Platform Profile}

The core supporting platform of the integrated management system uses three layer system structures, namely access layer, business layer and data layer. The architecture of the three layers ensures the efficient operation of the system, and can focus on the resources and access users. Platform will show the user, business process and business data that are abstracted in presentation layer, business logic layer and data layer, there are divided into three relatively independent units, to realize the low coupling between layers and the data processing of high cohesion in the layers. Core application supporting platform is based on J2EE architecture, the architecture has standard open database interface, building a business as the center of the system and achieving unified data storage and access mechanisms, and providing integrated service accessing interface $[5,6]$. Improving the platform and expanding its unified standards have strong flexibility. The system architecture is fully using the oriented object software reuse technology to solve the specific problems based on the field of web application with a strong reusability, which provides the overall specification framework for the rapid design and development of meteorological department enterprise application.

Multi-tier system.With the expansion of government system function, post deployment modules easily appear no unified planning and no uniform standard, so it is necessary to unified standards, unified planning application support platform as the key basis facilities for integrated management system. The main idea of application support platform extracts the public data and the corresponding data processing service of relevant functional departments from government business processes, which not only shorten development cycle, but also reduce development costs, and making the improvement and expansion of the application system always follow the unified standard [7].Its multi tier architecture is more prominent system planning in the software engineering supporting platform.

(1) The data layer is divided into two layers that are data sub layer and service sub layer. Data layer is the basis of the system. Data layer service sub layer provides uniform and standard atomic services for the business layer, which is used to shield business data storage, organization and access details, and realizes the full sharing of business data; business layer must through atomic services to access business data.

(2) The business logic of the business layer goes through the atomic services of the data layer to access the business data, a group of business logic processes can achieve specific business functions. Business layer provides a unified business logic process to achieve business logic in the business layer.

(3) The application supporting platform is estamblished based on J2EE, the multi-layer architecture system provides advanced and convenient component oriented method to architecture system in the platform, which will have strong secondary development and the ability to adapt to change.

Structural features. The application support platform is composed of the development and application framework, which includes three levels of service layer, application layer and presentation layer. System logical architecture is shown in Figure 1, the suite goes through definition process, organizational structure, form and the data source properties, and then the use of visualization tools carry out configuration, including office automation, online approval, leadership decision support, portals, business systems and integration and integrated office application system to carry on the rapid development, assembly and deployment, to effectively shorten the project launch cycle, improve project quality, reduce project risks for the user, and ensure that users have a lower total cost of ownership (TCO). The platform system has the following characteristics [8]:

(1) Based on the J2EE technology architecture, this system support scross platform, cross regional, cross network access running;

(2) Supporting WebService, SOAP, XML, WFMC, etc.;

(3) Providing a flexible two development tool, to support new business, new features' the rapid development capabilities;

(4) Tool design provides a wealth of visual customization tools and engines;

(5) Providing advanced programmer level of rich application components and application modules; 
(6) Having a highly personalized, user-friendly interface and operation mode;

(7) Based on the open architecture design, this system provides a rich interface, and it is convenient with the comprehensive integration and reuse of the third party system.

\section{The Construction of the Platform Key Technology}

In order to ensure all the government works in the smooth flow to efficient operation, we has detailed planning at the initial stage of the construction, that is in line with the management mode of the provincial bureau and can reflect the separation tube, to further through the scientific process management and improve the existing management problems [9]. The overall characteristic of integrated management system achieves the basic functions of e-government, combined with the actual requirements of the meteorological department, to carry out innovation in the form, content and function. Its essence is the innovation of Fujian meteorological department in the meteorological administration information and management science.

E-government is the combination of government affairs management and information technology, and it is the project that leads the government management innovation. Although the construction of basic information modernization equipment is more fully, the construction of information technology is not a co-ordination of planning, which causes one of the main problems of Information Island. Therefore the application supporting platform of the provincial bureau e-government carries out construction, implementation and development as the key technology. The main ideas of application support platform refines the public data and corresponding data processing services of the relevant functional departments from the bureau business process, in order to build a business as the center of the system, achieving unified data storage and access mechanisms, mining a data for the same data and providing integrated service access interface. The application of supporting platform is not only to shorten the development cycle, but also to improve the application system and expansion as well as follows the unified standards, at the source to eliminate the information island. The application supporting platform designed by Provincial Bureau contains the application development, expansion supporting platform and application framework, which provides a service - oriented layer.

UML modeling. UML (Unified Modeling Language) is also known as unified modeling language standard modeling language, and it is a support model and software system development of graphical language, to provide the modeling and visualization support for all phases of software development. UML is a kind of modeling language which is well defined, easy to express, powerful and widely used, it is integrated into the new ideas, new methods and new technologies in the field of software engineering, and it has a wide application area. The most commonly used is the model establishment of the software system, but also can be used to describe non software system, such as mechanical system, enterprise or business processes and complex data information system etc.. UML modeling technology goes through application supporting platform, it is not only concerned with the system functional requirements of structure and behavior, but also relates to system performance, easy to understand and reuse and non-functional requirements, which uses model view, structure model view, behavior view model to describe the platform structure. When the application-supporting platform is in the extended function or reuse module, the UML's view structure can guarantee the system's quality and the normal operation of the internal operation mechanism.

The system authorization mechanism in the application of support platform is a good embodiment of UML technology. Authorization function contains the permission inheritance function, which can ensure authorization mechanism between different user groups and user to carry on copy and paste, but also can choose the output user group or user permissions list table, to complete group of users or users the entrance management of the service function. Based on the role access control, the directory server saves the ACL information and record user or roles, in which the UML structure model view is shown in Figure 1. 


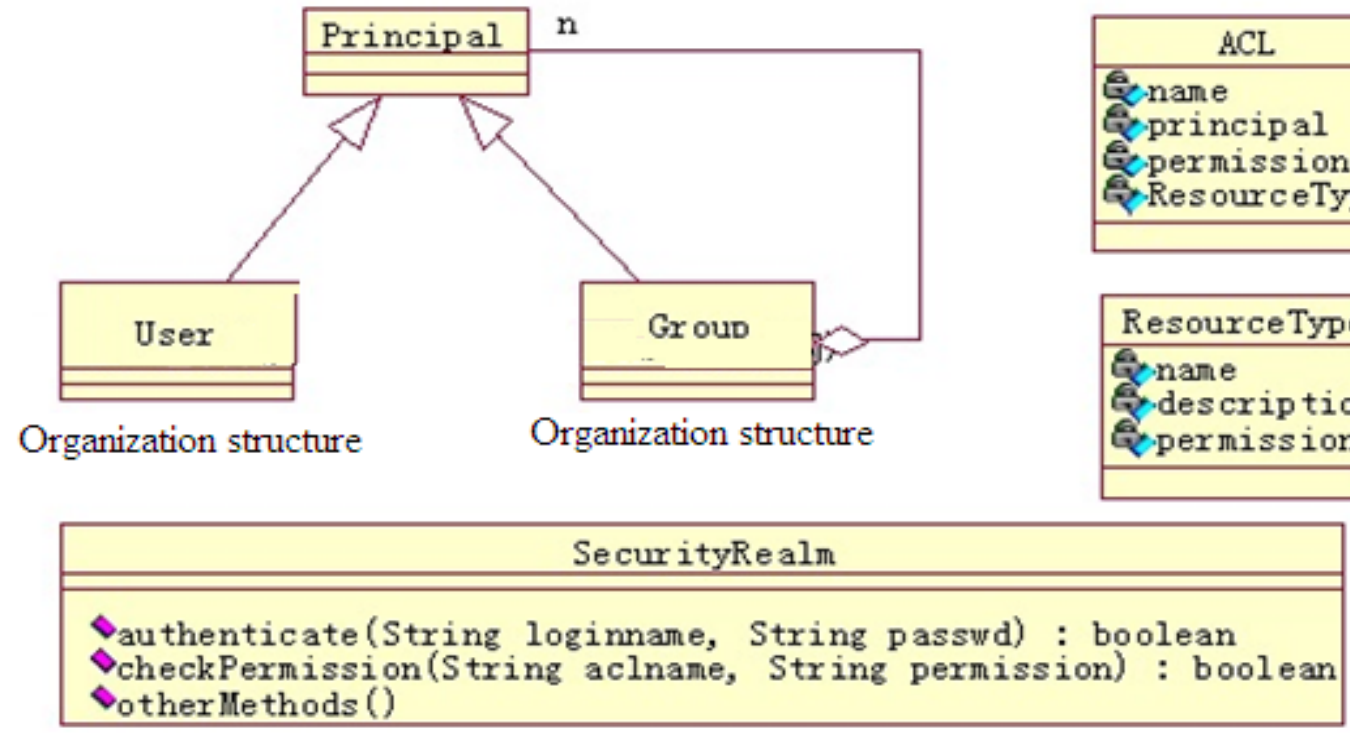

Fig.1 UML structure model view

Authority can be based on the user, but also can be based on role. The authority of a user is direct own authorization and the sum of belongs role permissions.

Workflow technology. Workflow is business plan abstract, summary and description between work flow and its operational procedures. The logic or rules of the organization are represented in the computer and the calculation is carried out before and after work in the work flow. Workflow is to achieve a business goal and to use a computer can automatically transfer documents, information or tasks according to a predetermined rule. Based on the characteristics of the e-government system, the application support platform is particularly dependent on the workflow technology. Workflow technology is the core technology of the construction process.

Workflow in the process of document management is divided into three stages: process modeling, process simulation, process improvement or optimization. Process modeling is a clear and formal method to represent the process of different abstract levels; reliable model is based process analysis; process simulation is to find the existing flow problems so as to provide guidance for the process improvement; these three stages are the process of continuous evolution, and it is an indispensable platform for the integrated management system. In the work flow of three stage, there are constantly interactions, in the application supporting platform making the document flow configuration has the graphic workflow definition, workflow engine, workflow supervision, management tools, work list and other necessary modules, they support sub workflow, application integration, overtime, work flow variables and basic function, and has high performance, reliability, transaction processing ability and failure recovery capability, which can not only support the development of electronic official document flow, but also will be able to support other e-government business process development and integration, to further the formation process of the application support platform. Workflow engine is the most important in the workflow technology, there are two parts, including data model and control model. Data model includes mechanism model and information model, the mechanism model describes the relationship between the enterprise and the department organization; the information model is defined as the control data used in the workflow engine. Through data model, it is convenient to describe the business rules, dependence on activity, tasks assignment etc.

Data exchange. The data exchange technology of application support platform provides the ability of distributed deployment system and other systems interconnection for the system, the data transfer and exchange between distributed systems can be realized by data exchange technology. Interchange format uses the universal XML format, it is developed standard data by the W3C, because XML has structured, extensible and platform independent, data format is transmitted in the HTTP protocol and widely applied between different platforms and different systems to carry on data exchange. The exchange data structure of the supporting platform is divided into three levels, and there are the system description layer, the process control layer and the data control layer. The data 
control layer is the specific implementation of the data exchange, including the exchange control core and database driven part; data exchange control core is the key, including a variety of data access operations, data upload and download process, safety control and error control. As a kind of data carrier, XML has not realized the preservation of data, so the security problem becomes an urgent need to solve the problem. If the system uses SSL or TSL encryption protocol, it can only achieve the file level encryption, it cannot be used encryption for elements. In order to be a good solution the data exchange when the data is stolen, the data exchange will do a the data encryption.

\section{Summary}

This paper proposes the core application platform of Fujian province meteorological bureau integrated management information system based on J2EE architecture. To achieve the province's government management, this paper develops the high availability, security, scalability and reliability core support platform, it is proved by theory and practice that the key techniques of UML modeling can quickly realize the specification design and inheritance of the system function, the system can not only well meet the needs of software engineering and visualization process configuration system, but also can be faster and more flexible process configuration to provide better support the environment for government work, the application of data compression and encryption technology ensures good information safety and safe operation of the system. The complex systems of a computing model and running environment multi-layer system structure set a variety of different application function, in the administrative it supports the one of Fujian meteorological department e-government, information feedback, administrative management process standardization, scientific online office electronic administrative affairs, and administrative affairs sharing. In the platform macro significance, management achieves the macro management of government affairs and affairs, it really does best supporting platform of Fujian e-government system, which provides a good core applications for e-government system further integration meteorological data.

\section{References}

[1] W.P. W., S.T. W., W.M. W.. A lightweight EAI architecture based on J2EE, Spring and Hibernate. Application of computer system, 2005(11):38-41.

[2] X.B. T., S.Y. C.. Research and application of MVC design pattern in J2EE architecture. Journal of Xihua University (NATURAL SCIENCE EDITION), 2013,26 (2): 16-17.

[3] H. Z.. Research and application of role access control based on J2EE architecture. Wuhan: Wuhan University of Technology, 2014: 15-20.

[4] J. X.. Design and implementation of educational administration management information system based on J2EE architecture. Chengdu: University of Electronic Science and technology, 2013: 2-11.

[5] Y.B. Z.. Design and implementation of medical information service platform based on J2EE framework. Beijing: Beijing Jiao Tong University, 2014: 34-40.

[6] D.C. Z.. The .Net and J2EE applications integrated based on Web services. Guangxi Institute of Computer Science in 2014. Guangxi Guilin: Guangxi computer society, 2014:40-44.

[7] H.M. G., D. X., Z.T. L.. The test research progress based on UML activity diagram. Computer science, 2014,35(2): 267-271.

[8] K.F. X.. Modeling research and application of e-commerce system based on UML.

Anhui: HeFei University of Technology, 2013: 2-10. 